\title{
Gene-gene interaction between the monoamine oxidase A gene and solute carrier family 6 (neurotransmitter transporter, noradrenalin) member 2 gene in anorexia nervosa (restrictive subtype)
}

Ruth E Urwin* ${ }^{* 1,6}$, Bruce H Bennetts ${ }^{2,8}$, Bridget Wilcken ${ }^{3,8}$, Basiliki Lampropoulos ${ }^{4}$, Peter JV Beumont ${ }^{5,6}$, Janice D Russell ${ }^{6}$, Sue L Tanner ${ }^{7}$ and Kenneth P Nunn ${ }^{1,6}$

\footnotetext{
${ }^{1}$ Department of Psychological Medicine, The Children's Hospital at Westmead, Westmead, NSW, Australia;

${ }^{2}$ Department of Molecular Genetics, The Children's Hospital at Westmead, Westmead, NSW, Australia; ${ }^{3}$ Department of Biochemical Genetics \& Newborn Screening, The Children's Hospital at Westmead, Westmead, NSW, Australia; ${ }^{4}$ Adolescent Medical Unit, Westmead Hospital, Westmead, NSW, Australia; ${ }^{5}$ Department of Psychiatry, Royal Prince Alfred Hospital, Camperdown, NSW, Australia; ${ }^{6}$ Discipline of Psychological Medicine, University of Sydney, NSW, Australia; ${ }^{7}$ Wesley Private Hospital, Ashfield, NSW, Australia; ${ }^{8}$ Discipline of Paediatrics and Child Health, University of Sydney, NSW, Australia
}

We earlier found an association between anorexia nervosa (AN) restrictive subtype (AN-R) and an inserted sequence within the NETPPR, a polymorphic region located in the promoter of the solute carrier family 6 (neurotransmitter transporter, noradrenalin) member 2 (SLC6A2) gene. To further examine the noradrenergic system in AN-R we performed an association study with a functional polymorphism (MAOAUVNTR) in the promoter of the monoamine oxidase A (MAOA) gene. Since monoamine oxidase $A$ metabolises noradrenalin, a positive association with the MAOA gene would be biologically plausible. The transmission disequilibrium test and 95 trios/duos (AN-R females + biological parents) showed the main effect of the longer, more transcriptionally active form of the MAOA-uVNTR (MAOA-L) to be statistically non-significant (McNemar's $\chi^{2}=1.4, \mathrm{df}=1, P=0.238$, odds ratio: $1.4,95 \% \mathrm{Cl} 0.8-2.7$ ). A case-control approach supported this finding. We then stratified the MAOA-uVNTR TDT data according to the (a) NETpPR genotype of the AN-R females, and (b) NETpPR allele transmitted from NETpPR-S4/L4 heterozygous mothers. In both cases, contingency table analysis revealed previously unreported genegene interaction between the MAOA and SLC6A2 genes $(P=0.019$ and 0.019 , respectively). Receiving an MAOA-L allele more than doubles the risk for developing $A N-R$, conditional on an individual also being a NETpPR-L4 homozygote (stratum-specific odds ratio: $2.4,95 \% \mathrm{Cl} 1.1-6.0$ ). These results suggest important involvement of the noradrenergic system in the biological underpinnings of AN-R. European Journal of Human Genetics (2003) 11, 945-950. doi:10.1038/sj.ejhg.5201077

Published online 24 September 2003

Keywords: eating disorders; catecholamines; gene-gene interaction

*Correspondence: RE Urwin, Department of Psychological Medicine, The Children's Hospital at Westmead, Locked Bag 4001, Westmead, NSW, 2145, AUSTRALIA. Tel: +1 16129845 2005; Fax: + 16129845 2009; E-mail: RuthU@chw.edu.au

Received 24 March 2003; revised 4 June 2003; accepted 9 July 2003
Introduction

DSM-IV $^{1}$ anorexia nervosa (AN) is an eating disorder in which body weight is maintained at less than $85 \%$ of 
normal, there is amenorrhoea in females, an intense fear of gaining weight, and a disturbed perception of one's shape and/or weight. There are two types of AN based on whether sufferers control their weight by restricting food intake only (AN-R) or by purging (AN-BP). A genetic component is suggested in AN. ${ }^{2,3}$ Past and current speculation is that the serotonergic system is the main neurotransmitter system involved in AN due to increased cerebrospinal fluid (CSF) levels of the serotonin metabolite 5-hydroxyindoleacetic acid (5-HIAA) in long-term weight-restored patients compared to normals ${ }^{4}$ and the clinical efficacy of selective serotonin reuptake inhibitors in individuals after weight gain. ${ }^{5}$

Monoamine oxidase A (MAOA; EC 1.4.3.4) metabolises serotonin and noradrenalin, and to a lesser extent dopamine. MAOA is encoded by a gene at Xp11.23. ${ }^{6}$ The functional MAOA-upstream variable number of tandem repeats (MAOA-uVNTR) polymorphism ${ }^{7,8}$ in the MAOA gene promoter commonly consists of 3 (3-allele) or 4 (4allele) copies of a 30-bp sequence, or rarely 2 (2-allele) or 3 copies plus the first 18 -bp of the same $30-b p$ sequence (3aallele), or 5 copies (5-allele). The 3a-allele and 4 -allele are transcribed more efficiently than the shorter 3-allele. In male skin fibroblast cultures, the 4-allele results in increased MAOA activity relative to the 3 -allele. ${ }^{9}$ The effect of the 2-allele on transcription is unknown and of the 5allele debatable. ${ }^{7,8}$

We recently demonstrated an association between AN-R and the L4 allele and L4/L4 genotype of an insertion(L4)/deletion(S4) polymorphism in the NETpPR. ${ }^{10}$ The NETpPR is a polymorphic region first described by us ${ }^{10}$ within the promoter region of the solute carrier family 6 (neurotransmitter transporter, noradrenalin) member 2 (SLC6A2) gene located on chromosome $16 .{ }^{11}$ SLC6A2 is responsible for reuptake of noradrenalin into the presynaptic neuron where it can be metabolised by MAOA. As CSF and blood noradrenalin levels are low in long-term weight-restored $\mathrm{AN}$ patients compared to normals $^{12,13}$ and MAOA metabolises noradrenalin, we hypothesised that the MAOA-uVNTR was involved in susceptibility to AN-R.

Psychiatric disorders (eg AN) are complex diseases thought to be due to the combined effect of environmental factors and multiple genes. Each gene contributes to the genetic component of the disorder independently and/or through gene-gene interaction with one or a number of other genes. ${ }^{14}$ As SLC6A2 and MAOA are the major proteins responsible for clearing noradrenalin from the synaptic cleft ${ }^{15}$ we believed the probability of gene-gene interaction between the MAOA and SLC6A2 genes in AN-R was likely to be higher relative to two genes chosen randomly.

Here we report the first association study of the MAOA gene and $\mathrm{AN}$, and the first investigation of gene-gene interaction between the MAOA and SLC6A2 genes.

\section{Materials and methods Subjects}

The 95 unrelated families ( 87 trios, 8 duos) (91 Caucasian: 4 East Asian) in this study were recruited from Sydney and Melbourne, Australia and included the 87 AN-R trios from our earlier study, ${ }^{10}$ plus 8 AN-R duos recruited later. Each trio consisted of one DSM-IV AN-R female plus both of her biological parents. Each duo consisted of one DSM-IV AN-R female plus her biological mother. Ethics approval had been gained from the appropriate ethics committees and all participants gave written informed consent (parents signed for those under 14 years). The 95 consecutive consenting AN-R females were enrolled into the study as inpatients $(n=85)$ if they were medically compromised, or as outpatients $(n=10)$. All patients fulfilled criteria for DSM-IV AN-R except that the amenorrhoea criterion was waived for the prepubertal females. Patients were excluded from the study if both parents were not likely to be available for testing. To ensure that patients were consistent in keeping their weight low by restricting food intake and not by purging, diagnosis of AN-R was achieved through using several "layers" of assessment over a period of time including structured clinical interviews with the patient and relatives, and case note searches by a researcher who was not involved in patient care. The original diagnosis was made (blind to genotype) by eating disorder specialists and confirmed with $95 \%$ consensus by one of three psychiatrists (KPN, JDR, PJVB) also blind to genotype, by using all previously accumulated data and through a structured clinical interview. Where any diagnostic ambiguity existed, KPN personally reviewed the case notes to confirm or reject the diagnosis. Ages and body mass index (BMI) were recorded during assessment. BMI was determined from weight and height measured by hospital staff, the weight criterion for DSM-IV AN-R being based on the minimum BMI recorded. At minimum BMI, all patients aged 11-15 years weighed less than or equal to their age and sex specific 5th percentile BMI cutoff as determined in an Australian population study. ${ }^{16}$ Above age 15 years a BMI of 17.5 was the upper limit. ${ }^{17}$ Of the 95 patients, 31 were under 15 years of age (mean age $13.61 \pm 1.23$ years) at their minimum BMI (mean minimum BMI $14.35 \pm 1.59$ ) reached at a mean of $0.89 \pm 0.57$ years after onset of $A N-R$, and 64 were aged 15 years and over (mean age $17.89 \pm 3.13$ years) at minimum BMI (mean minimum BMI 14.66 \pm 1.69 ) reached at a mean of $2.17 \pm 2.03$ years after onset of ANR. The young age of our patients precluded setting a required period of time for patients to meet a diagnosis of AN in the absence of purging to be considered AN-R. However, as shown above, patients under 15 years of age had suffered from AN-R for an average of 1 year, while older patients had suffered from AN-R for an average of 2 years. As patients with AN-R may cross over to AN-BP or bulimia nervosa within the first few years of onset of AN-R we must 
therefore acknowledge the possibility of heterogeneity in our sample.

\section{Molecular genetic methods}

A $10 \mathrm{ml}$ EDTA venous blood sample was collected from the AN-R females and their parents. Genomic DNA was extracted from the blood using standard methods. ${ }^{18}$ NETpPR genotypes of the 87 AN-R trios in the current report had been determined in our earlier study ${ }^{10}$ where the NETpPR genotyping methodology is described. NETpPR genotypes of the additional 8 AN-R duos recruited later were determined in the current study. NETpPR genotypes of the total 95 AN-R females in the current study were 63 L4/L4, 29 S4/L4, and 3 S4/S4.

The MAOA-uVNTR was PCR-amplified using previously published primer sequences ${ }^{19}$ : forward primer MAOAFor2 $5^{\prime}$-CCCAGGCTGCTCCAGAAAC and reverse primer MAOARev2 5'-GGACCTGGGCAGTTGTGC, but with modified amplification conditions, as follows. PCR reactions were carried out in $10 \mu \mathrm{l}$ volumes containing $10 \mathrm{~mm}$ Tris$\mathrm{HCl} \mathrm{pH} \mathrm{8.3,} 50 \mathrm{~mm} \mathrm{KCl}, 1.5 \mathrm{~mm} \mathrm{MgCl}_{2}, 0.5 \mu \mathrm{M}$ of each primer, $200 \mu \mathrm{M}$ of each dNTP, $0.25 \mathrm{U}$ Ampli-Taq Gold (Applied Biosystems, Foster City, CA, USA), and 50 ng DNA template. The GeneAmp PCR System 9700 (Applied Biosystems) was used for thermal cycling: $95^{\circ} \mathrm{C}$ for $10 \mathrm{~min}$; 30 cycles of $95^{\circ} \mathrm{C}$ for $30 \mathrm{~s}, 67-52.5^{\circ} \mathrm{C}$ for $30 \mathrm{~s}$ (annealing temperature decreased by $0.5^{\circ} \mathrm{C} /$ cycle over the 30 cycles), $72^{\circ} \mathrm{C}$ for $40 \mathrm{~s} ; 10$ cycles of $95^{\circ} \mathrm{C}$ for $30 \mathrm{~s}, 52^{\circ} \mathrm{C}$ for $30 \mathrm{~s}, 72^{\circ} \mathrm{C}$ for $40 \mathrm{~s}$; and finally $72^{\circ} \mathrm{C}$ for $10 \mathrm{~min}$. PCR products were separated according to size on $8 \%$ polyacrylamide gels. DNA fragments corresponding to the 3-repeat (209-bp), rare 3a-repeat (227-bp), 4-repeat (239-bp), and rare 5-repeat (269-bp: seen in 2 fathers in the current study) were identified by comparison to a molecular weight DNA standard, and a known control containing the 2-repeat (179-bp), 3-repeat, and 4-repeat alleles. The ABI Prism Big Dye Terminator Ready Reaction Kit with Ampli-Taq DNA polymerase, FS (Applied Biosystems) and the ABI Prism 310 Genetic Analyser (Applied Biosystems) were used to confirm the DNA sequence of a representative homozygote for each of the different MAOA-uVNTR alleles detected. Maternal MAOA-uVNTR allele data were (transmitted:nontransmitted) MAOA-L (63:55) and MAOA-S (32:40). MAOAuVNTR genotypes of the AN-R females were $50 \mathrm{MAOA}-\mathrm{L} / \mathrm{L}$, $36 \mathrm{MAOA}-\mathrm{S} / \mathrm{L}$, and $9 \mathrm{MAOA}-\mathrm{S} / \mathrm{S}$.

\section{Statistical analysis}

The transmission disequilibrium test (TDT) ${ }^{20}$ measured association in the family-based sample (ie trios/duos). The TDT (McNemar's $\chi^{2}$ ) uses data derived from parents who are heterozygous and detects preferential transmission of one allele to affected children. In the TDT, the nontransmitted parental alleles act as the control group thereby diminishing spurious associations resulting from ethnic differences between cases and controls. We used the $\chi^{2}$ test for the case-control analysis and for measuring Hardy-Weinberg equilibrium. Gene-gene interaction was assessed with Fisher's exact tests for $2 \times 2$ and $\mathrm{R} \times \mathrm{C}$ Tables. The required significance level was set at 0.025 after Bonferroni correction as two independent tests were performed: full sample (Stage 1) and stratified sample (Stage 2). Two-sided $P$-values were used. Case-control power calculations were performed using SPSS SamplePower.

\section{Results}

The MAOA-uVNTR genotypes determined in the current study include those of the 87 AN-R trios (AN-R female + biological parents) involved in our earlier study ${ }^{10}$ in which only their NETpPR genotypes were determined. An additional 8 AN-R duos (AN-R female + biological mother) recruited after our earlier study ${ }^{10}$ had their MAOA-uVNTR and NETpPR genotypes determined in the current study (ie. total of 95 families).

\section{MAOA-uVNTR association study}

The 95 trios/duos were genotyped for the MAOA-uVNTR. All mothers in the 'duos' were informative for their MAOAuVNTR genotype (ie. no duo was composed of 2 heterozygous individuals). Therefore no duos were discarded which would have introduced bias. ${ }^{21}$ We used the TDT for data analysis. Since the MAOA gene is on the X-chromosome, a father has only one allele which is always transmitted to his daughter (ie no non-transmitted control allele) but never to his son. The fathers' genotyping results were therefore not included in the analysis but were used only to determine which alleles were transmitted from the mothers in the 'trios'. For data analysis, the MAOA-uVNTR alleles were collapsed into two groups based on transcriptional efficiency ${ }^{7,8}$ using the same nomenclature as in earlier reports, ${ }^{7,8}$ (a) the shorter 3-allele [MAOA-Short (MAOA-S)] and longer (b) 3a-allele +4 -allele [MAOA-Long (MAOA-L)]. No 2-alleles or 5-alleles were present in the maternal alleles. Pre-study power calculations ${ }^{22}$ showed that with our 95 trios/duos we had $80 \%$ power to detect an association if MAOA-L was considered as the high-risk allele $(\alpha=0.05$, genotypic relative risk (GRR) of MAOA-S/ $\mathrm{L}=2.6$, MAOA-L allele frequency $=0.65$ reported earlier ${ }^{23}$ ). We had $80 \%$ power to detect an association if MAOA-S was considered as the high risk allele $(\alpha=0.05$, GRR of MAOA$\mathrm{S} / \mathrm{L}=2.3$, MAOA-S allele frequency $=0.35$ reported ear$\left.\mathrm{lie}^{23}\right)$. Genotypes of mothers and their AN-R daughters were in Hardy-Weinberg equilibrium: $\chi^{2}=0.13, \mathrm{df}=1$, $P=0.719$, and $\chi^{2}=0.45, \mathrm{df}=1, P=0.504$, respectively. TDT analysis of the combined strata from Table 1 showed that MAOA-L was transmitted more often to the daughters: 27 MAOA-L vs 19 MAOA-S. However, this finding was not statistically significant (McNemar's $\chi^{2}=1.4, \quad \mathrm{df}=1$, $P=0.238$, odds ratio: $1.4,95 \%$ CI $0.8-2.7$ ). We were able to confirm our findings using a case-control approach as 
Table 1 MAOA-uVNTR TDT data from 46 heterozygous mothers stratified by AN-R daughter's NETpPR genotype

\begin{tabular}{|c|c|c|c|}
\hline \multirow{2}{*}{$\begin{array}{l}\text { Daughter's } \\
\text { NETpPR } \\
\text { Genotype }\end{array}$} & \multicolumn{3}{|c|}{ Allele transmitted from MAOA-S/ } \\
\hline & $\mathrm{L}$ & $S$ & Ratio $(95 \% \mathrm{Cl})$ \\
\hline L4/L4 & 22 & 9 & $2.4(1.1-6.0)$ \\
\hline S4/L4 & 5 & 7 & $0.7(0.2-2.6)$ \\
\hline S4/S4 & 0 & 3 & $0.0(0-2.4)$ \\
\hline Combined & 27 & 19 & $1.4(0.8-2.7)$ \\
\hline
\end{tabular}

L4/L4 vs S4/L4 vs S4/S4 Fisher's 2-sided exact test for $\mathrm{R} \times \mathrm{C}$ Tables: $P=0.019$.

genotyping data were available from 453 Caucasian female controls drawn from the general Australian population. ${ }^{23}$ The genotype frequencies of the female controls were in Hardy-Weinberg equilibrium: $\chi^{2}=0.2, \mathrm{df}=1, \quad P=0.688$. Comparing allele data from the AN-R females (ie. transmitted maternal alleles listed in 'Molecular genetic methods') and controls ${ }^{23}$ gave a similar result $\left(\chi^{2}=2.6, \mathrm{df}=1\right.$, $P=0.104$, MAOA-L odds ratio: $1.3,95 \%$ CI $0.9-1.9)$ to that obtained with the TDT. Comparing genotypes of the AN-R females (listed in 'Molecular genetic methods') and controls $^{23}$ showed increased frequency of MAOA-L/L in AN-R females but this finding was also not significant (MAOA-L/ $\mathrm{L}$ vs $\mathrm{MAOA}-\mathrm{S} / \mathrm{L}+\mathrm{MAOA}-\mathrm{S} / \mathrm{S}: \chi^{2}=2.8, \mathrm{df}=1, \quad P=0.095$, odds ratio: $1.5,95 \%$ CI $0.9-2.3)$.

\section{MAOA gene-SLC6A2 gene interaction study}

Only one gene-gene interaction was explored (ie. MAOA$\mathrm{uVNTR}$ with NETpPR). We previously observed that AN-R is strongly associated with both the NETPPR-L4 allele and NETpPR-L4/L4 genotype ${ }^{10}$ and it appeared from the 2 -sided $P$-values above, ranging from 0.095 to 0.238 , that MAOA-L was a possible susceptibility allele for AN-R. We hypothesised a priori that conditional on the NETpPR-L4 homozygous genotype or L4 allele being transmitted, MAOA-L would also be preferentially transmitted to AN-R females. Gene-gene interaction was assessed by stratifying the MAOA-uVNTR TDT data from Stage 1, based on the AN-R female NETpPR genotypes (Table 1) to form a $3 \times 2$ contingency table. The top, middle, and bottom strata were composed of MAOA-uVNTR TDT data from mothers of the NETpPR-L4/L4 AN-R females, NETpPR-S4/L4 AN-R females and NETpPR-S4/S4 AN-R females, respectively. Visual inspection of the contingency table data showed that association in the top stratum was in the opposite direction to that in the middle and bottom strata. We compared the strata using a Fisher's exact test for $\mathrm{R} \times \mathrm{C}$ Tables. Since this comparison was significant $(P=0.019)$ gene-gene interaction was suggested and it was appropriate to report stratum-specific odds ratios rather than a summary measure. An additive interaction model was suggested within the tabulated data. MAOA-L was prefer-
Table 2 MAOA-uVNTR TDT data from 20 mothers who were both NETpPR-S4/L4 and MAOA-S/L heterozygotes, stratified by the NETpPR allele transmitted to their $20 \mathrm{AN}-\mathrm{R}$ daughters

\begin{tabular}{|c|c|c|c|}
\hline \multirow{2}{*}{$\begin{array}{l}\text { NETPPR allele } \\
\text { transmitted }\end{array}$} & \multicolumn{2}{|c|}{ MAOA-uVNTR allele transmitted } & \multirow{2}{*}{$\begin{array}{l}\text { MAOA-L Odds } \\
\text { Ratio }(95 \% \mathrm{Cl})\end{array}$} \\
\hline & MAOA-L & MAOA-S & \\
\hline L4 & 10 & 2 & $5.0(1.1-47)$ \\
\hline & 2 & 6 & $0.3(0.03-1.9)$ \\
\hline Combined & 12 & 8 & $1.5(0.6-4.2)$ \\
\hline
\end{tabular}

L4 vs S4 Fisher's 2-sided exact test: $P=0.019$.

entially transmitted in the NETpPR-L4/L4 stratum (odds ratio: 2.4 , 95\% CI 1.1-6.0), but neither in the NETpPR-S4/ L4 stratum (odds ratio: $0.7,95 \%$ CI $0.2-2.6$ ) nor in the NETpPR-S4/S4 stratum (odds ratio: 0.0, 95\% CI 0.0-2.4). NETpPR alleles appeared to have an additive effect on susceptibility. The NETPPR genotype distribution was dependent upon which MAOA-uVNTR allele was transmitted.

In the gene-gene interaction analysis above, both parents contributed alleles to the NETpPR genotypes of AN-R females. However, only mothers contributed MAOA$\mathrm{uVNTR}$ alleles to the analysis. We considered that unequal input of genotype data from mothers and fathers suggested a possible source of bias, which we addressed by removing the fathers' NETpPR data. MAOA-uVNTR TDT data from the mothers $(n=20)$ who were both NETpPR-S4/L4 and MAOA-S/L heterozygotes were stratified based on their NETpPR allele transmitted (Table 2). All MAOA-S/L heterozygous mothers were informative for their NETpPR genotype and none were discarded. Fisher's exact test showed a significant difference between the strata $(P=0.019)$ again suggesting gene-gene interaction. MAOA-L was preferentially transmitted in the NETpPR-L4 stratum (odds ratio: $5.0,95 \%$ CI $1.1-47$ ) but not in the NETpPR-S4 stratum (odds ratio: 0.3 , 95\% CI 0.03-1.9).

The required level of significance was set at 0.025 after Bonferroni correction for two independent tests: full sample (Stage 1) and stratified sample (Stage 2). We considered that further use of the Bonferroni correction within our stratified analyses (ie. two gene-gene interaction analyses) was too conservative for two reasons. Firstly, gene-gene interaction was biologically plausible as MAOA and SLC6A2 are the major proteins responsible for clearing noradrenalin from the synaptic cleft. ${ }^{15}$ Secondly, we knew that the NETpPR L4 allele and L4/L4 genotype are significantly associated with $\mathrm{AN}-\mathrm{R}^{10}$ and felt that MAOA$\mathrm{L}$ would be preferentially transmitted to AN-R females based on the results from Stage 1 of the current study. Therefore one hypothesis,specified a priori, involving one independent comparison was appropriate. Nevertheless, as both $P$-values in the stratified analysis were 0.019 (just 
below the set 0.025 level of significance) our findings could be a false positive and therefore require replication in a much larger study.

\section{Discussion}

In this paper, we have described the first association study of the MAOA gene and $\mathrm{AN}$, and the first investigation of gene-gene interaction between the MAOA and SLC6A2 genes. We have also provided data that suggest gene-gene interaction between the MAOA and SLC6A2 genes in AN-R. To summarise, AN-R females who are NETpPR L4 homozygotes and/or have the NETpPR-L4 allele preferentially transmitted to them, are significantly more likely to also receive MAOA-L, suggesting gene-gene interaction between the MAOA and SLC6A2 genes in AN-R. The gene-gene interaction we have observed cannot be explained by physical closeness of the genes as the MAOA and SLC6A2 genes are located on different chromosomes. However, functional variants in linkage disequilibrium with one or both of the polymorphisms studied here may be contributing to susceptibility to develop AN-R.

If a gene's independent contribution to a disorder is small, which appears to be the case with the MAOA-uVNTR in the MAOA gene in AN-R females, it is often difficult to identify this contribution as impractical numbers of subjects are required to retain statistical power. To detect our observed odds ratio of 1.4 with MAOA-uVNTR TDT data (using our observed MAOA-L frequency of 0.62 for mothers in the calculations) would require 622 trios to allow $80 \%$ power as only the mothers' results can be used. ${ }^{22}$ A case-control study using our observed MAOA-L frequency of 0.72 for $A N-R$ females and the reported frequency of 0.65 for normal females ${ }^{23}$ would require 692 AN-R females and 692 normal controls. The effect of a second susceptibility gene (in this case, the MAOA gene) may become apparent, however, when gene-gene interactions with other genes are analysed. ${ }^{24,25}$ We have illustrated the effectiveness of this approach in our gene-gene interaction analysis, and have also shown how the relationship of the associated susceptibility loci (in this case the MAOA-uVNTR and NETpPR) may be easily characterised in a contingency table.

Although evidence of a statistical interaction as we report here does not necessarily map directly onto a biological interaction, true gene-gene interactions nonetheless must have a biological basis ${ }^{26}$ and the proteins encoded by the MAOA and SLC6A2 genes work together to clear excess noradrenalin from the synaptic cleft. SLC6A2 returns noradrenalin to the presynaptic neuron where MAOA metabolises it. ${ }^{15}$ MAOA activity or noradrenalin reuptake by SLC6A2 are inhibited by several drugs used to treat depression and anxiety. ${ }^{27}$ Depression and anxiety are often present before, during, and post-AN. ${ }^{28-30}$ One study showed that the antidepressant serotonin and noradrenalin reuptake inhibitor, venlafaxine, may be beneficial for patients with atypical $\mathrm{AN}^{31}$ in which weight remains just above the diagnostic threshold for AN or amenorrhoea is absent. ${ }^{1}$ Low CSF and blood noradrenalin levels observed in long-term weight-restored AN patients compared to normals ${ }^{12,13}$ may be explained by increased MAOA activity which would integrate well with our finding of association of AN-R with MAOA-L as this variant results in increased MAOA activity. ${ }^{9}$

Further work is required to investigate gene-gene interaction between other genes involved in clearing noradrenalin from the synaptic cleft to progressively elucidate the pathophysiology involved in AN-R. Investigating genegene interactions with catechol-O-methyltransferase (COMT) would be important. Although playing a minor role compared to MAOA, COMT is the second most important enzyme involved in clearing noradrenalin from the synaptic cleft. Additionally, the COMT gene allele which is responsible for increased COMT activity has already shown an independent association with $\mathrm{AN}^{32}$

Prior to our previous study ${ }^{10}$ it appeared that serotonin was the major neurotransmitter involved in AN. ${ }^{4,5}$ However, integrating the results from our earlier study ${ }^{10}$ with the findings described here, suggest important involvement of the noradrenergic system in the biological underpinnings of AN-R.

\section{Acknowledgements}

This study was supported by the Children's Hospital Fund Small grant SGS9808 and donations from the Estate of the Late Charlotte Ross and from Anne Shaw Kennedy. We thank Prof Derek Gordon from the Laboratory of Statistical Genetics, Rockefeller University, USA, for statistical advice and are grateful to Drs Wiley and Carpenter at The Children's Hospital at Westmead for the use of their laboratories. We appreciate the recruitment of study participants at 'Footprints of Angels', Northside Clinic, Royal Prince Alfred Hospital, The Children's Hospital at Westmead, Wesley Private Hospital, and Westmead Hospital. We specially thank the families for participating.

\section{References}

1 American Psychiatric Association. Diagnostic and Statistical Manual of Mental Disorders, 4th edn. Washington, DC: American Psychiatric Association, 1994.

2 Wade TD, Bulik CM, Neale M, Kendler KS: Anorexia nervosa and major depression: shared genetic and environmental risk factors. Am J Psychiatry 2000; 157: 469-471.

3 Klump KL, Miller KB, Keel PK, McGue M, Iacono WG: Genetic and environmental influences on anorexia nervosa syndromes in a population-based twin sample. Psychol Med 2001; 31: 737-740.

4 Kaye WH, Gwirtsman HE, George DT, Ebert MH: Altered serotonin activity in anorexia nervosa after long-term weight restoration. Does elevated cerebrospinal fluid 5hydroxyindoleacetic acid level correlate with rigid and obsessive behavior? Arch Gen Psychiatry 1991; 48: 556-562.

5 Kaye WH, Nagata T, Weltzin TE et al: Double-blind placebocontrolled administration of fluoxetine in restricting- and 
restricting-purging-type anorexia nervosa. Biol Psychiatry 2001; 49: $644-652$.

6 Lan NC, Heinzmann C, Gal A et al: Human monoamine oxidase A and B genes map to Xp11.23 and are deleted in a patient with Norrie disease. Genomics 1989; 4: 552-559.

7 Sabol SZ, Hu S, Hamer D: A functional polymorphism in the monoamine oxidase A gene promoter. Hum Genet 1998; 103: $273-279$.

8 Deckert J, Catalano M, Syagailo YV et al: Excess of high activity monoamine oxidase A gene promoter alleles in female patients with panic disorder. Hum Mol Genet 1999; 8: 621-624.

9 Denney RM, Koch H, Craig IW: Association between monoamine oxidase A activity in human male skin fibroblasts and genotype of the MAOA promoter-associated variable number tandem repeat. Hum Genet 1999; 105: 542-551.

10 Urwin RE, Bennetts B, Wilcken B et al.: Anorexia nervosa (restrictive subtype) is associated with a polymorphism in the novel norepinephrine transporter gene promoter polymorphic region. Mol Psychiatry 2002; 7: 652-657.

11 Brüss M, Kunz J, Lingen B, Bönisch H: Chromosomal mapping of the human gene for the tricyclic antidepressant-sensitive noradrenaline transporter. Hum Genet 1993; 91: 278-280.

12 Kaye $\mathrm{WH}$, Jimerson DC, Lake CR, Ebert $\mathrm{MH}$ : Altered norepinephrine metabolism following long-term weight recovery in patients with anorexia nervosa. Psychiatry Res 1985; 14: $333-342$.

13 Pirke KM, Kellner M, Philipp E, Laessle R, Krieg JC, Fichter MM: Plasma norepinephrine after a standardized test meal in acute and remitted patients with anorexia nervosa and in healthy controls. Biol Psychiatry 1992; 31: 1074-1077.

14 Stoltenberg SF, Burmeister M: Recent progress in psychiatric genetics-some hope but no hype. Hum Mol Genet 2000; 9: 927-935.

15 Carson RP, Robertson D: Genetic manipulation of noradrenergic neurons. J Pharmacol Exp Ther 2002; 301: 410-417.

16 Harvey PWJ, Althaus M: The distribution of body mass index in Australian children aged 7-15 years. Aust J Nutr Diet 1993; 50: $151-153$

17 World Health Organisation. The ICD-10 Classification of Mental and Behavioral Disorders: Clinical Descriptions and Diagnostic Guidelines. Geneva, Switzerland: World Health Organisation, 1992, pp 176-177.

18 Miller SA, Dykes DD, Polesky HF: A simple salting out procedure for extracting DNA from human nucleated cells. Nucleic Acids Res 1988; 16: 1215-1215.
19 Samochowiec J, Lesch KP, Rottmann M et al: Association of a regulatory polymorphism in the promoter region of the monoamine oxidase A gene with antisocial alcoholism. Psychiatry Res 1999; 86: 67-72.

20 Spielman RS, McGinnis RE, Ewens WJ: Transmission test for linkage disequilibrium: the insulin gene region and insulindependent diabetes mellitus (IDDM). Am J Hum Genet 1993; 52: 506-516.

21 Curtis D, Sham PC: A note on the application of the transmission disequilibrium test when a parent is missing. Am J Hum Genet 1995; 56: 811-812, (letter).

22 Risch N, Merikangas K: The future of genetic studies of complex human diseases. Science 1996; 273: 1516-1517.

23 Jorm AF, Henderson AS, Jacomb PA et al: Association of a functional polymorphism of the monoamine oxidase A gene promoter with personality and psychiatric symptoms. Psychiatr Genet 2000; 10: 87-90.

24 Ritchie MD, Hahn LW, Roodi N et al: Multifactor-dimensionality reduction reveals high-order interactions among estrogenmetabolism genes in sporadic breast cancer. Am J Hum Genet 2001; 69: 138-147.

25 Zubenko GS, Hughes 3rd HB, Stiffler JS: D10S1423 identifies a susceptibility locus for Alzheimer's disease in a prospective, longitudinal, double-blind study of asymptomatic individuals. Mol Psychiatry 2001; 6: 413-419.

26 Botto LD, Khoury MJ: Commentary: facing the challenge of geneenvironment interaction: the two-by-four table and beyond. $\mathrm{Am} J$ Epidemiol 2001; 153: 1016-1020.

27 Owens MJ: Molecular and cellular mechanisms of antidepressant drugs. Depress Anxiety 1996-97; 4: 153-159.

28 Deep AL, Nagy LM, Weltzin TE, Rao R, Kaye WH: Premorbid onset of psychopathology in long-term recovered anorexia nervosa. Int J Eat Disord 1995; 17: 291-297.

29 Kaye WH: Persistent alterations in behavior and serotonin activity after recovery from anorexia and bulimia nervosa. Ann N Y Acad Sci 1997; 817: 162-178.

30 Kaye WH, Klump KL, Frank GKW, Strober M: Anorexia and bulimia nervosa. Annu Rev Med 2000; 51: 299-313.

31 Ricca V, Mannucci E, Paionni A et al: Venlafaxine versus fluoxetine in the treatment of atypical anorectic outpatients: a preliminary study. Eat Weight Disord 1999; 4: 10-14.

32 Frisch A, Laufer N, Danziger Y et al: Association of anorexia nervosa with the high activity allele of the COMT gene: a family-based study in Israeli patients. Mol Psychiatry 2001; 6: $243-245$. 